\title{
The X-Trials: Neural Correlates of an Inhibitory Control Task in Children and Adults
}

\section{Citation}

Davis, Elysia Poggi, Jacqueline Bruce, Kelly Snyder, and Charles A. Nelson. 2003. "The X-Trials: Neural Correlates of an Inhibitory Control Task in Children and Adults." Journal of Cognitive Neuroscience 15 (3) (April): 432-443.

\section{Published Version}

doi:10.1162/089892903321593144

\section{Permanent link}

http://nrs.harvard.edu/urn-3:HUL.InstRepos:13497071

\section{Terms of Use}

This article was downloaded from Harvard University's DASH repository, and is made available under the terms and conditions applicable to Other Posted Material, as set forth at http:// nrs.harvard.edu/urn-3:HUL.InstRepos:dash.current.terms-of-use\#LAA

\section{Share Your Story}

The Harvard community has made this article openly available.

Please share how this access benefits you. Submit a story.

\section{Accessibility}




\title{
The X-Trials: Neural Correlates of an Inhibitory Control Task in Children and Adults
}

\author{
Elysia Poggi Davis, Jacqueline Bruce, Kelly Snyder, \\ and Charles A. Nelson
}

\begin{abstract}
Event-related potentials (ERPs) were used to examine developmental differences between adults and 6-year-old children in the neural processes involved in an inhibitory control task. Twenty adults and 21 children completed a task that required them to selectively respond to target stimuli while inhibiting responses to equally salient non-target stimuli. Because this task had been previously studied using functional magnetic resonance imaging (fMRI), the relation
\end{abstract}

\section{INTRODUCTION}

The control of action and thought plays an important role in the organization of human behavior. Inhibitory control is the capacity for active inhibition or modulation of a prepotent response and is viewed as falling under the general rubric of executive functions. Studies of inhibitory control have found that areas of the frontal cortex play a role in inhibition of a prepotent motor response (Mishkin, 1964). Poor performance on tasks involving inhibition of a motor response has been found to be associated with dysfunction in the frontal lobes (van Leewen et al., 1998). Furthermore, animals and humans with frontal lobe lesions exhibit deficits in the ability to inhibit a motor response (Chao \& Knight, 1995; Iversen \& Mishkin, 1970). Additionally, studies using positron emission tomography (PET) and functional magnetic resonance imaging (fMRI) have indicated that a cortical network of frontal areas, including the anterior cingulate cortex (ACC) and the orbital prefrontal cortex (OFC), subserve the ability to inhibit responses (Carter et al., 2000; Casey et al., 1997; Pardo, Pardo, Janer, \& Raichle, 1990).

The ability to inhibit prepotent responses has been found to improve with age. It has been argued that maturation of the prefrontal cortex (PFC) accounts for the developmental improvements seen in inhibitory control (Dempster, 1992). Diamond (1989) and Diamond and Goldman-Rakic (1989) have found that frontal lobe development is necessary for both human and

University of Minnesota between the fMRI and ERP findings was informally examined. The results indicate that latency and amplitude of the P3 differentiated the different types of trials. However, the pattern of event-related neural activity differed for adults and children. These results, which suggest that adults and children may be using different processes to perform this task, have implications for the interpretation of the previous fMRI findings.

monkey infants to succeed on tasks requiring the inhibition of prepotent responses. Moreover, dysregulation in the development of these frontal regions has been implicated in a significant number of childhood psychiatric disorders, such as attention deficit-hyperactivity disorder (ADHD), that seem to involve a failure of the ability to inhibit responses (van Leewen et al., 1998; Gorenstein, Mammato, \& Sandy, 1989; Chelune, Ferguson, Koon, \& Dickey, 1986).

The Continuous Performance Task (CPT) is one task that has frequently been used to assess inhibitory control. A version of this task, designed by Casey et al. (1997), has been used in fMRI studies to assess the neural substrates involved in the inhibition of a motor response. The task requires the execution of an anticipated motor response or its active inhibition. Participants are primed to respond to target stimuli and are then required to inhibit their response to equally salient non-target stimuli. More specifically, participants are asked to press a button as quickly as possible in response to the presentation of every letter, except for the letter "X," without making mistakes. In this version of the task, there are two blocks of trials. Block 1 consists of the first 42 trials containing 100\% target stimuli (i.e., Block 1 go trials). These trials prime participants to respond to the target stimuli. Block 2 is the response inhibition condition. It consists of 42 trials containing 50\% target stimuli (i.e., Block 2 go trials) and $50 \%$ non-target stimuli (no-go trials).

In a neuroimaging study employing this task, activation of the inferior frontal gyrus, middle frontal gyrus, OFC, and ACC was observed for both adults and 7- to 
12-year-old children in the response inhibition condition (Casey et al., 1997). Thus, adults and children showed greater activation of these regions during Block 2, which contained 50\% target and 50\% nontarget stimuli, as compared to Block 1, which contained $100 \%$ target stimuli. Furthermore, children showed greater activation of these regions than adults did. Activation of two regions, the ACC and the OFC, was associated with performance. Greater activation of the ACC was associated with a greater number of false alarms (i.e., pressing the button to a non-target stimulus) whereas greater activation of the OFC was associated with fewer false alarms.

fMRI is an extremely useful technique that has vastly improved our understanding of the neural structures involved in cognitive processing, but there are limitations inherent to this measure. First, largely because of the time course of the hemodynamic response, fMRI has poor temporal resolution on the order of seconds. Event-related potentials (ERPs), on the other hand, provide information on the order of milliseconds regarding the mental chronometry of information processing (Keifer, Marzinzik, Weisbrod, Scherg, \& Spitzer, 1998; van Leewen et al., 1998). Thus, this method can be used to further our understanding of the neural processes involved in cognitive functioning. In contrast to fMRI, which tends to be most suitable for older children who can remain still for long periods of time, ERPs can be used with children of any age. Thus, it is an excellent tool by which to study developmental change. Collectively, whereas use of fMRI with the CPT has furthered our understanding of neural structures involved in the ability to inhibit a motor response, our understanding of temporal sequencing in the processing of the task is limited. Using fMRI, we are not able to determine whether the frontal structures found to be involved in this response inhibition task are responsible for different aspects of the processing of this task. Second, because of the block design used with this task in the previous fMRI study, it is not possible to differentiate between the neural processes involved in the go trials of Block 2 as compared to no-go trials of Block 2. Thus, although it is clear that greater inhibition is required in Block 2 than in Block 1, it is likely that different processes would be used to regulate the response to a go stimulus as compared to a no-go stimulus in Block 2. ERPs may be beneficial in improving our understanding of processes involved in the decision to respond as compared to processes involved in inhibiting a motor response.

Previous ERP studies with similar CPT tasks have found greater amplitude and later latency of the N2 and $\mathrm{P} 3$ during conditions requiring the inhibition of a motor response (Fallgatter \& Strik, 1999; van Leewen et al., 1998; Eimer, 1993). During these tasks, the N2 has been thought to reflect the decision to withhold or inhibit a motor response (Jackson, Jackson, \& Roberts, 1999) and the $\mathrm{P} 3$ reflects the relevance of the stimuli to the participant (Tekok-Kilic, Schucard, \& Schucard, 2001; Keifer et al., 1998). It has been suggested that the amplitude of the P3 is greater for no-go stimuli than go stimuli when the response preparation is high (Cohen, Porjesz, Begleiter, \& Wang, 1997). This may be related to the attention (Overtoom et al., 1998) or magnitude of inhibition (Cohen et al., 1997) elicited by the stimuli.

It was a goal of this study to use ERPs to examine the task-relevant neural processes of an inhibitory control task previously studied using fMRI and to compare these processes in adults and children. Based on the results from the fMRI study (Casey et al., 1997), it was predicted that greater activation would be seen for both adults and children on trials that required response inhibition (i.e., no-go trials). Second, it was predicted that children would show greater activation on these trials than adults. Additionally, based on prior ERP studies, it was predicted that greater amplitude of the $\mathrm{N} 2$ and the $\mathrm{P} 3$ would be seen on trials requiring inhibition of a motor response (Fallgatter \& Strik, 1999; van Leewen et al., 1998; Eimer, 1993).

Whereas it is important to understand neural processes involved in a cognitive task, it is equally important to understand factors related to individual differences in performance on such tasks. A second goal of this study, then, was to investigate factors that might predict behavioral performance on this task. There are two relevant components of behavior, speed and accuracy. There is evidence from tasks similar to the one used in this study that greater amplitude of the N2 (Falkenstein, Hoormann, \& Hohnsbein, 1999; Verbaten et al., 1994) and P3 (Verbaten et al., 1994) is associated with greater accuracy. Furthermore, increases in processing speed, as indicated by latency of the N2, were also found to be related to increased accuracy (Falkenstein et al., 1999). However, previous research has suggested that for tasks that emphasize speed as well as accuracy, such as the task used in this study, reaction time and latency are not related (Donchin, Karis, Bashiore, Coles, \& Gratton, 1986).

In sum, there were several goals of the current study. The primary goal of this study was to use ERPs to examine the neural processes of an inhibitory control task previously studied using fMRI. Additionally, we intended to explore the relation between behavioral performance on this task and the relevant ERP components.

\section{RESULTS}

\section{Behavioral Data}

The number of errors and mean reaction times for the three different types of trials are displayed in Table 1. Comparisons were made between the adults' and children's performance during the different trials for both accuracy and speed. 
Table 1. Descriptive Statistics for Behavioral Data

\begin{tabular}{|c|c|c|c|c|c|c|}
\hline & \multicolumn{2}{|c|}{ Block 1 Go Trials } & \multicolumn{2}{|c|}{ Block 2 Go Trials } & \multicolumn{2}{|c|}{ No-Go Trials } \\
\hline & $M$ & $S D$ & $M$ & $S D$ & $M$ & $S D$ \\
\hline \multicolumn{7}{|c|}{ Number of Errors } \\
\hline Adults & 0.15 & 0.49 & 0.15 & 0.49 & 1.55 & 1.50 \\
\hline Children & 1.70 & 2.41 & 2.90 & 3.23 & 6.25 & 3.51 \\
\hline \multicolumn{7}{|c|}{ Reaction Time (msec) } \\
\hline Adults & 274.56 & 136.82 & 352.35 & 115.24 & & \\
\hline Children & 432.52 & 112.81 & 569.67 & 116.47 & & \\
\hline
\end{tabular}

$n=20$ for both adults and children.

To assess accuracy on the task, a $2 \times 3$ (Age $\times$ Trial) ANOVA with repeated measures on the last factor was computed. Adults were significantly more accurate than children, $F(1,38)=43.65, p<.001{ }^{1}$ Additionally, there was a main effect of trial for accuracy, $F(1.55,58.94)=$ $20.97, p<.001$. Post hoc tests revealed that participants were more accurate on the Block 1 go and Block 2 go trials than the no-go trials, $t(39)=-5.46, p<.001$ and $t(39)=-3.96, p<.001$, respectively. Furthermore, the age by trial interaction was significant, $F(1.55,58.94)=$ $5.35, p<.05$. Both adults and children were significantly more accurate on the Block 1 go and Block 2 go trials than the no-go trials, Block 1 go: $t(19)=-4.17, p<.005$ and $t(19)=-4.95, p<.001$; Block 2 go: $t(19)=-4.77$, $p<.001$ and $t(19)=-2.95, p<.01$. However, the difference in accuracy on Block 2 go trials as compared to no-go trials was greater for adults than children.

For reaction time, a $2 \times 2$ (Age $\times$ Trial) ANOVA with repeated measures on the last factor was computed. There was a main effect of age for reaction time, $F(1,38)=26.58, p<.001$. Adults had significantly shorter reaction times than children. There was also a main effect of trial for reaction time, $F(1,38)=86.93$, $p<.001$. Participants had faster reaction times on the Block 1 go trials than the Block 2 go trials. In addition, the age by trial interaction was significant, $F(1,38)=$ $6.63, p<.05$. Although both adults and children were significantly faster on the Block 1 go trials than the Block 2 go trials, $t(19)=-5.65, p<.001$ and $t(19)=-7.42$, $p<.001$, respectively, there was a greater difference between the reaction times on the Block 1 go trials in comparison to the Block 2 go trials for children.

In summary, adults were more accurate and had faster reaction times on the task than children. Additionally, adults and children made more errors on the no-go trials and demonstrated slower reaction times on the Block 2 go trials than the Block 1 go trials. These findings indicate that the task manipulation was effective and appears to be equivalent to the task used by Casey et al. (1997) in an fMRI environment.

\section{ERP Data}

The average peak amplitude and latency to peak of the three ERP components for each type of trial are displayed in Table 2. Figures 1 and 2 display the stimulustriggered ERPs for each condition at the analyzed frontal and parietal leads for adults and children.

\section{N2}

A $2 \times 3 \times 3($ Age $\times$ Trial $\times$ Lead $)$ ANOVA with repeated measures on last two factors was computed for peak amplitude of the N2. As seen in Figures 1 and 2, there was a main effect of age with children displaying greater negativity than adults, $F(1,39)=12.44, p<.005$. There was, however, no main effect of trial, $F(2,78)=.92, n s$, or lead, $F(2,78)=1.93$, ns. In addition, none of the interactions were significant. The absence of an amplitude difference for the no-go trials was surprising and contrasted with previous tasks involving response inhibition (Jackson et al., 1999; Overtoom et al., 1998; Eimer, 1993). A possible explanation for our failure to detect the expected amplitude differences in the N2 is that propagation of the $\mathrm{P} 3$ seems to have reduced our ability to detect the $\mathrm{N} 2$ in response to this task.

A $2 \times 3 \times 3$ (Age $\times$ Trial $\times$ Lead) ANOVA with repeated measures on last two factors was computed for latency to peak of the N2. There was a main effect of age with adults displaying shorter latency than children, $F(1,39)=106.68, p<.001$. There was no main effect of trial, $F(2,78)=.45$, $n s$, or lead, $F(2,78)=.71$, ns. However, the age by trial interaction was significant, $F(2,78)=11.22, p<.001$. Post hoc tests revealed that for children, the latency for the Block 1 go trials and the Block 2 go trials was shorter than the no-go trials, $t(20)=-3.38, p<.005$ and $t(20)=-2.47, p<.05$, respectively. Conversely, for adults, the latency for the no-go trials was shorter than the Block 1 go trials and the Block 2 go trials, $t(19)=-2.61, p<.05$ and $t(19)=$ $-3.43, p<.005$. It is possible, however, that the earlier 
latency for the no-go trials for adults may be an artifact of the proceeding positive component, which was larger for the no-go trials and may have obscured completion of the N2.

P3

A $2 \times 3 \times 3$ (Age $\times$ Trial $\times$ Lead) ANOVA with repeated measures on last two factors was computed for maximum amplitude of the P3. As seen in Figures 1 and 2, amplitude was greater for children as compared to adults, $F(1,38)=8.14, p<.01 .^{2}$ There was also a main effect of trial, $F(2,76)=16.53, p<.001$. However, the age by trial interaction was significant, $F(2,76)=4.66$, $p<.05$. Post hoc tests revealed that for adults, consistent with previous research (Fallgatter \& Strik, 1999; Eimer, 1993), amplitude for the no-go trials was greater than the Block 1 go trials and the Block 2 go trials, $t(19)=5.69, p<.001$ and $t(19)=3.66, p<.005$, respectively. In contrast, for children, amplitude for the Block 2 go and no-go trials was greater than the Block 1 go trials, $t(20)=3.48, p<.005$ and $t(20)=2.99$, $p<.01$, respectively. These results suggest that there may be a difference in the way that adults were processing this task as compared to children. Additionally, there was a main effect of lead, $F(2,76)=30.25, p<.001$, with greater amplitude at $\mathrm{Pz}$ as compared to $\mathrm{P} 3$ or $\mathrm{P} 4, t(39)=$ $6.36, p<.001$ and $t(40)=5.13, p<.001$, respectively. This finding indicates that this component is centrally distributed across parietal leads. The age by lead interaction was also significant, $F(2,76)=11.09, p<.001$. Post hoc tests revealed that the amplitude of the $\mathrm{P} 3$ was greater at $\mathrm{Pz}$ as compared to $\mathrm{P} 3$ or $\mathrm{P} 4$ for adults and children, P3: $t(18)=4.86, p<.001$ and $t(20)=6.28$, $p<.001$; P4: $t(19)=3.18, p<.01$ and $t(20)=5.28$, $p<.001$. However, the difference in amplitude at Pz as compared to $\mathrm{P} 3$ and $\mathrm{P} 4$ was greater for children.

A $2 \times 3 \times 3$ (Age $\times$ Trial $\times$ Lead) ANOVA with repeated measures on last two factors was computed for latency to peak of the P3. Adults displayed shorter latency than children, $F(1,38)=250.32, p<.001$. Additionally, the main effect of trial was significant, $F(2,76)=15.53, p<.001$. Latency to peak amplitude for the Block 1 go trials was shorter than the Block 2 go or no-go trials, $t(40)=4.86, p<.001$ and $t(40)=$ $4.27, p<.001$, respectively. Neither the main effect

Table 2. Descriptive Statistics for ERP Components

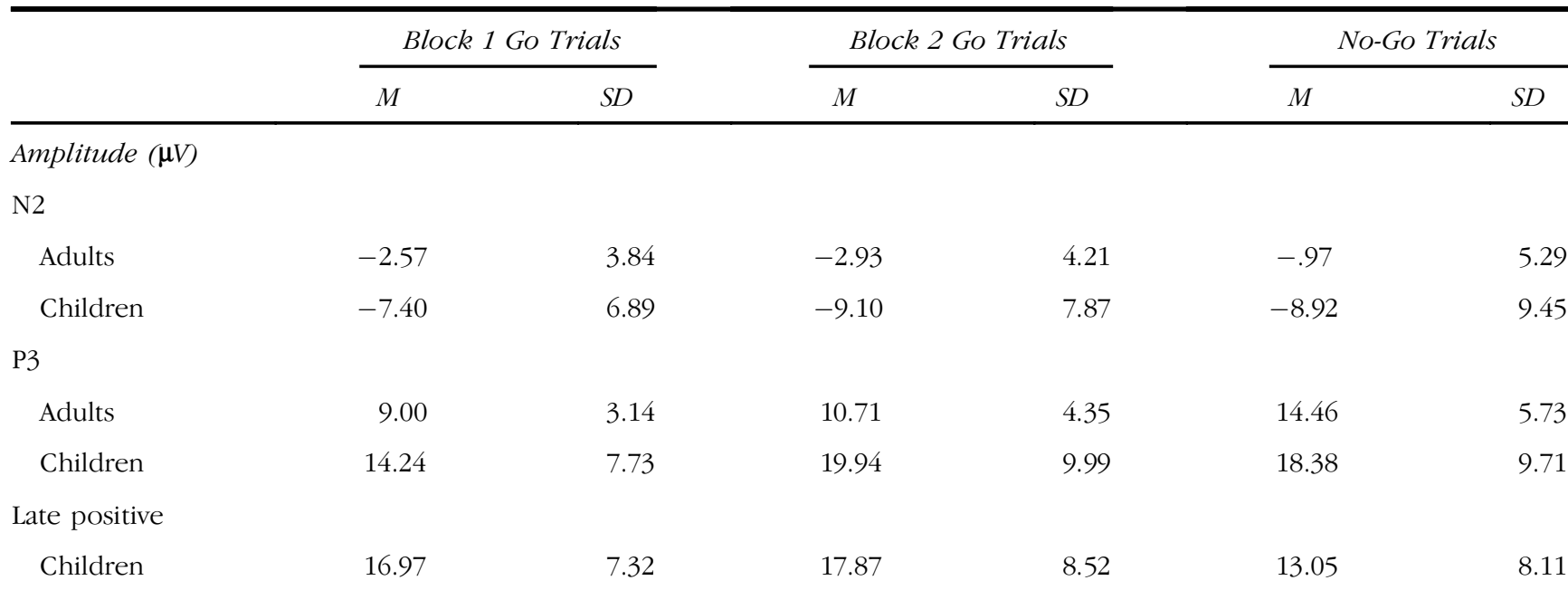

\section{Latency (msec)}

N2

\begin{tabular}{|c|c|c|c|c|c|}
\hline Adults & 373.42 & 59.09 & 367.25 & 48.40 & 332.33 \\
\hline
\end{tabular}

P3

\begin{tabular}{|c|c|c|c|c|c|c|}
\hline Adults & 373.58 & 58.02 & 488.50 & 68.51 & 494.50 & 47.38 \\
\hline
\end{tabular}

Late positive

$n=20$ for adults and $n=21$ for children.

Mean of data from Fz, F3, and F4 for N2 and late positive component, and mean of data from Pz, P3, and P4 for P3 


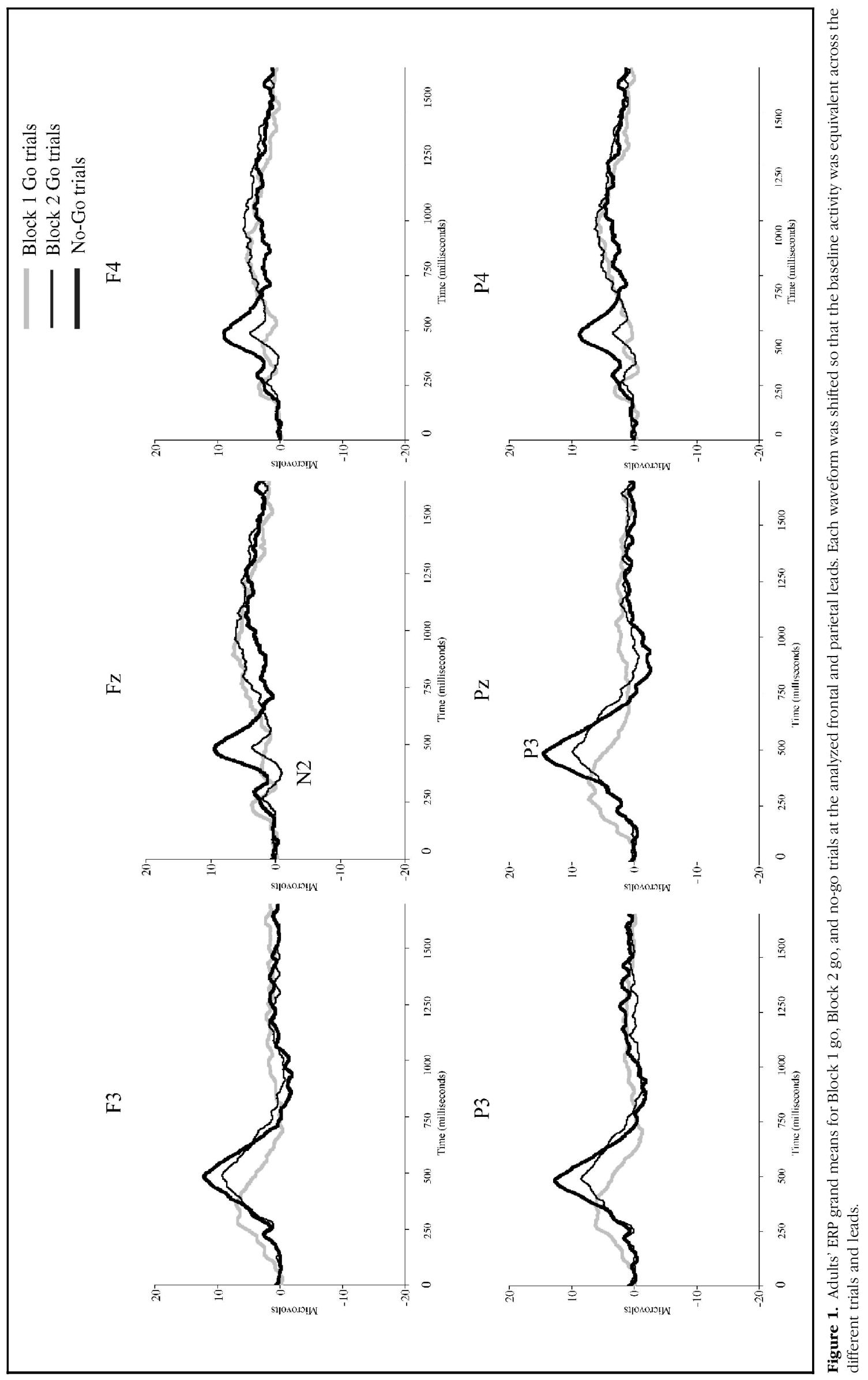




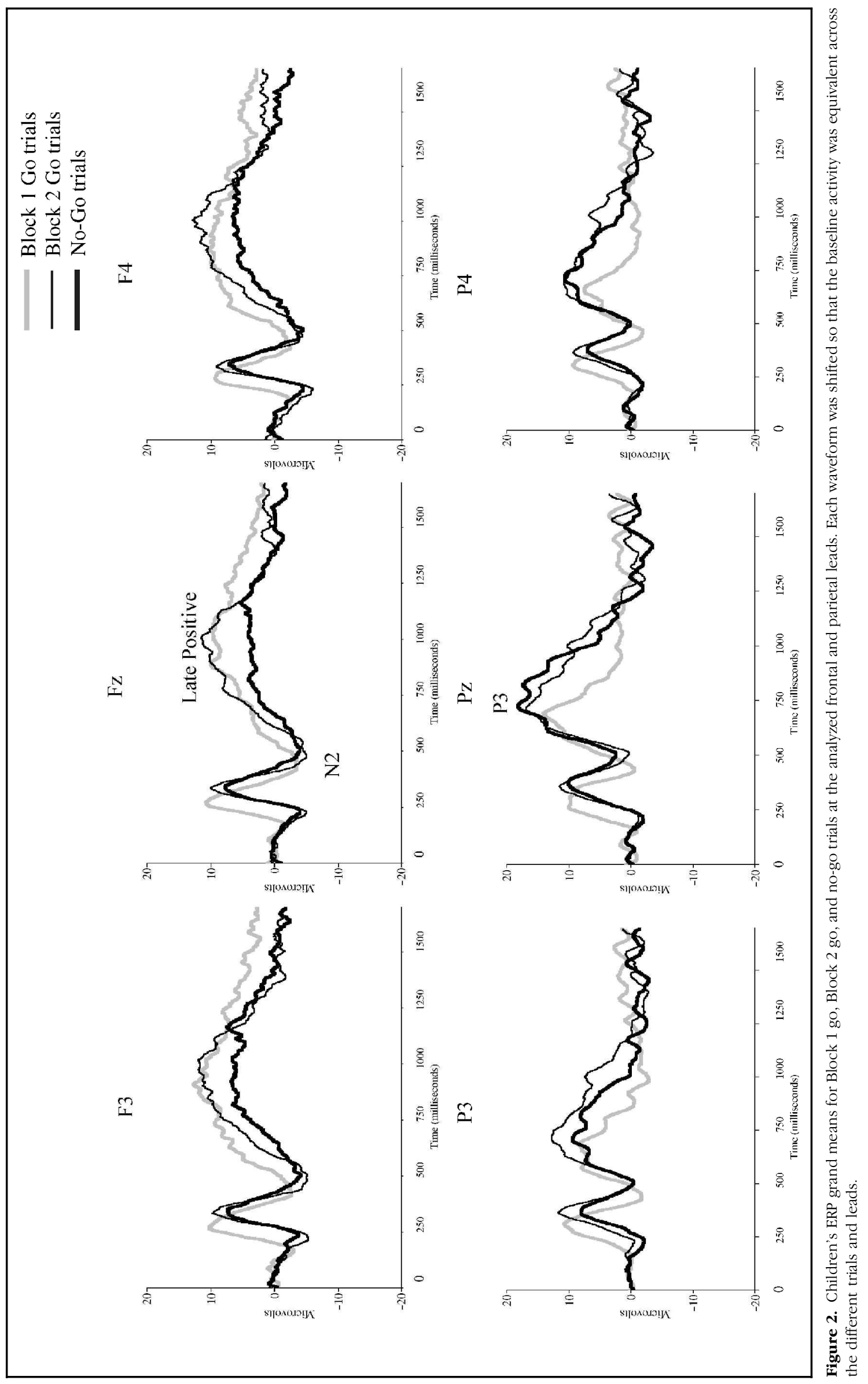

Davis et al. 437 
of lead, $F(2,76)=1.36, n s$, nor any of the interactions was significant.

\section{Late Positive Component}

Inspection of the ERP waveforms revealed that there was a late positive component for only the children. Thus, a $3 \times 3$ (Trial $\times$ Lead) repeated measures ANOVA was computed for maximum amplitude of this component, for children. There was a main effect of trial, $F(2,40)=$ $3.86, p<.05$. The amplitude was greater for the Block 1 go trials and the Block 2 go trials than the no-go trials, $t(20)=2.08, p<.05$ and $t(20)=2.23, p<.05$, respectively. Thus, for children, the late positive component differentiated the neural processes required by the no-go trials and the processes required by the Block 1 go trials and the Block 2 go trials. Additionally, there was a main effect of lead, $F(2,40)=3.91, p<.05$. Post hoc tests revealed that the amplitude was greater at $\mathrm{Fz}$ as compared to $\mathrm{F} 3$ or $\mathrm{F} 4, t(20)=2.54, p<.05$ and $t(20)=$ $2.70, p<.05$, respectively. This result indicates that the late positive component was centrally distributed across frontal leads for children.

A $3 \times 3$ (Trial $\times$ Lead) repeated measures ANOVA was computed for latency to peak. Neither the main effect of trial, $F(2,40)=1.95, n s$, nor lead, $F(2,40)=2.51, n s$, was significant. Additionally, the interaction between trial and lead was not significant. ${ }^{3}$

\section{Relation between Reaction Times and Latency of the ERP Components}

In order to understand the neural processes reflected by the ERP components, we explored the relation between the reaction times to the stimuli and the latency of the ERP components. As can be seen from the descriptive behavioral and ERP data (see Tables 1 and 2), the mean reaction time for the Block 1 go trials and the Block 2 go trials occurred at the beginning of the time window of the $\mathrm{P} 3$ for both adults and children. For adults, the reaction time to the stimuli occurred approximately 100- to 140 -msec earlier than the latency of the P3. However, the children's reaction time occurred approximately 220-270 msec earlier than the latency of the P3. The association between the reaction time to the stimuli and the latency of the ERP components was further explored using Pearson product correlations. To reduce the number of analyses, the mean of the analyzed frontal (i.e., Fz, F3, F4 for the N2 and late positive component) or parietal leads (i.e., Pz, P3, P4 for the P3) for each component was used instead of analyzing all the individual leads. In addition, because the reaction times and time windows of the ERP components were significantly different for the adults and children, the data were analyzed separately for adults and children. Reaction time was not significantly associated with the latency of any of the
ERP components for adults or children ( $r$ s ranged from -.40 to $.41, n s)$.

\section{Relation between False Alarms and ERP Components}

Finally, Pearson product correlations were used to investigate the association between false alarms and the ERP components. Due to the restricted variability in the accuracy of the adult participants, the analyses with false alarms were limited to child participants. The number of false alarms was not significantly related to the amplitude or latency of the ERP components ( $r$ s ranged from -.16 to $.31, n s)$.

\section{DISCUSSION}

Similar to previous research with this task (Casey et al., 1997), both adults and children made more errors and had a slower reaction time in Block 2, which involved response inhibition. The increased number of errors in Block 2 was primarily due to false alarms on the no-go trials. This illustrates that the task manipulation was effective. Despite the difference in age of the children and the context (i.e., from fMRI to ERP), the no-go trials elicited more errors. Furthermore, as was the case in the fMRI study, children made more errors and had slower reaction times than adults.

One goal of this study was to identify task-relevant neural processes involved in the inhibition of a motor response. In the case of the no-go stimuli, the prepared or prepotent response must be inhibited. Thus, the cognitive processes involved in inhibiting a motor response differentiate no-go trials from the Block 1 go and Block 2 go trials. However, both the no-go and the Block 2 go trials involved cognitive processes that differ from those involved in the Block 1 go trials. During Block 1, the participants develop an automatic pattern of responding to the stimuli presentation. This presumably requires minimal executive processing and decision making. In contrast, in Block 2, the participant must withhold their automatic response to the stimuli presentation in order to evaluate the stimuli and make a decision whether to execute or inhibit a response. Thus, there may be two types of inhibition involved in this task: (1) inhibition of the motor response until the stimulus has been evaluated for Block 2 trials and (2) inhibition of the motor response following stimulus evaluation for no-go trials.

Examination of the data suggests that the neural processes involved in this task differ for adults and children. As illustrated in Figure 3, greater amplitude of the P3 differentiated the no-go trials from the Block 1 go and Block 2 go trials for adults. In contrast, children displayed similar amplitude for the no-go and Block 2 go trials at parietal leads. Furthermore, the amplitude of the $\mathrm{P} 3$ for both the no-go and Block 2 
Figure 3. The Laplacian maps of the P3 at 440 msec for adults and at 765 msec for children.

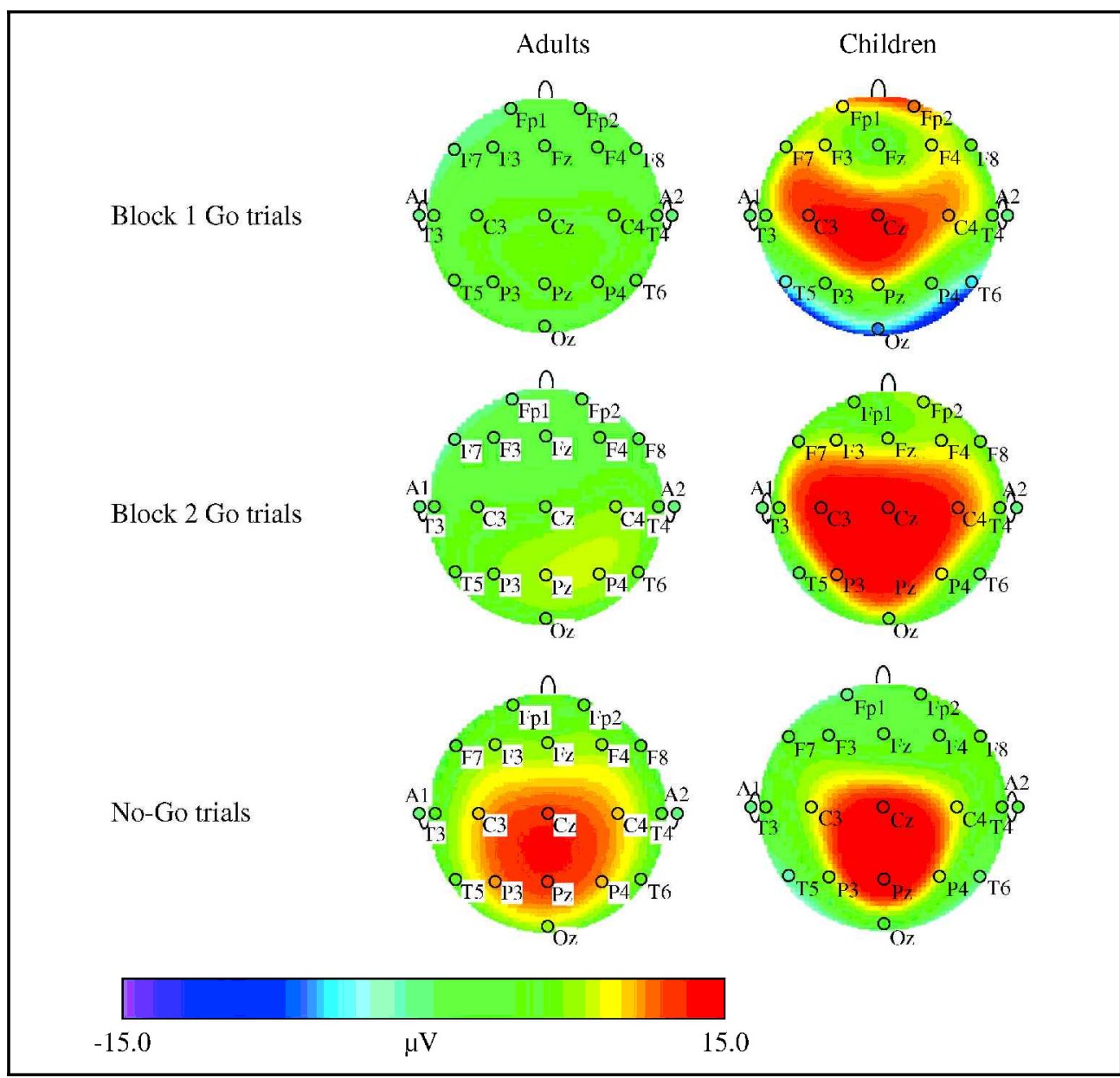

go trials was greater than the amplitude for the Block 1 go trials for children.

The reason for the divergent pattern of results for the P3 for adults and children is not clear from this study. One possibility may be that, although the $\mathrm{P} 3$ reflects a similar cognitive process for adults and children, children deployed this cognitive resource for both the Block 2 go and no-go trials and adults did not. It has been proposed that the amplitude of the $\mathrm{P} 3$ at parietal sites reflects the degree of task relevance or attention to the stimuli (Tekok-Kilic et al., 2001; Keifer et al., 1998; Overtoom et al., 1998). For adults, greater amplitude of the P3 for the no-go trials may suggest that these trials were more relevant and required more processing resources than the other trials. In contrast, children displayed similar amplitude for no-go and Block 2 go trials, indicating that these trials may have been equally relevant and required equivalent attentional resources. Alternatively, the divergent pattern of results may indicate that the P3 reflects different cognitive processes in adults and children. For example, it may be that for children the $\mathrm{P} 3$ is related to an early control process involved in withholding an automatic response in order to evaluate the stimuli during Block 2. In contrast, for adults, the $\mathrm{P} 3$ may be related to a later control process involved in inhibiting an incorrect response to the no-go trials. As noted above, the most plausible explanation for the divergent pattern of results for adults and children is not clear from the current study. Clearly, further research is required to explicate this finding.

Another goal of this study was to informally examine the relation between the ERP data and fMRI data collected with this task. In the fMRI study, greater activation of frontal regions was found in Block 2, which contained both go and no-go trials, for both adults and children. Furthermore, children displayed greater activation than adults (Casey et al., 1997). This was interpreted as reflecting developmental differences in cognitive processes required while performing this task. Because of the block design used in this fMRI study, go and no-go trials in Block 2 could not be examined separately. The ERP results with the task enhance the interpretations of the fMRI findings because of the ability to examine Block 2 go and no-go trials separately. The PFC activation found might be comparable to the $\mathrm{P} 3$ observed in the ERP data. As reported, for adults, the amplitude of this component was greater for the no-go trials while for children the Block 2 go and no-go trials showed greater amplitude than the Block 1 go trials. This suggests that, for adults, the no-go trials may have accounted for the greater activation seen in Block 2 in the fMRI study. For children, however, both the go and the no-go trials may 


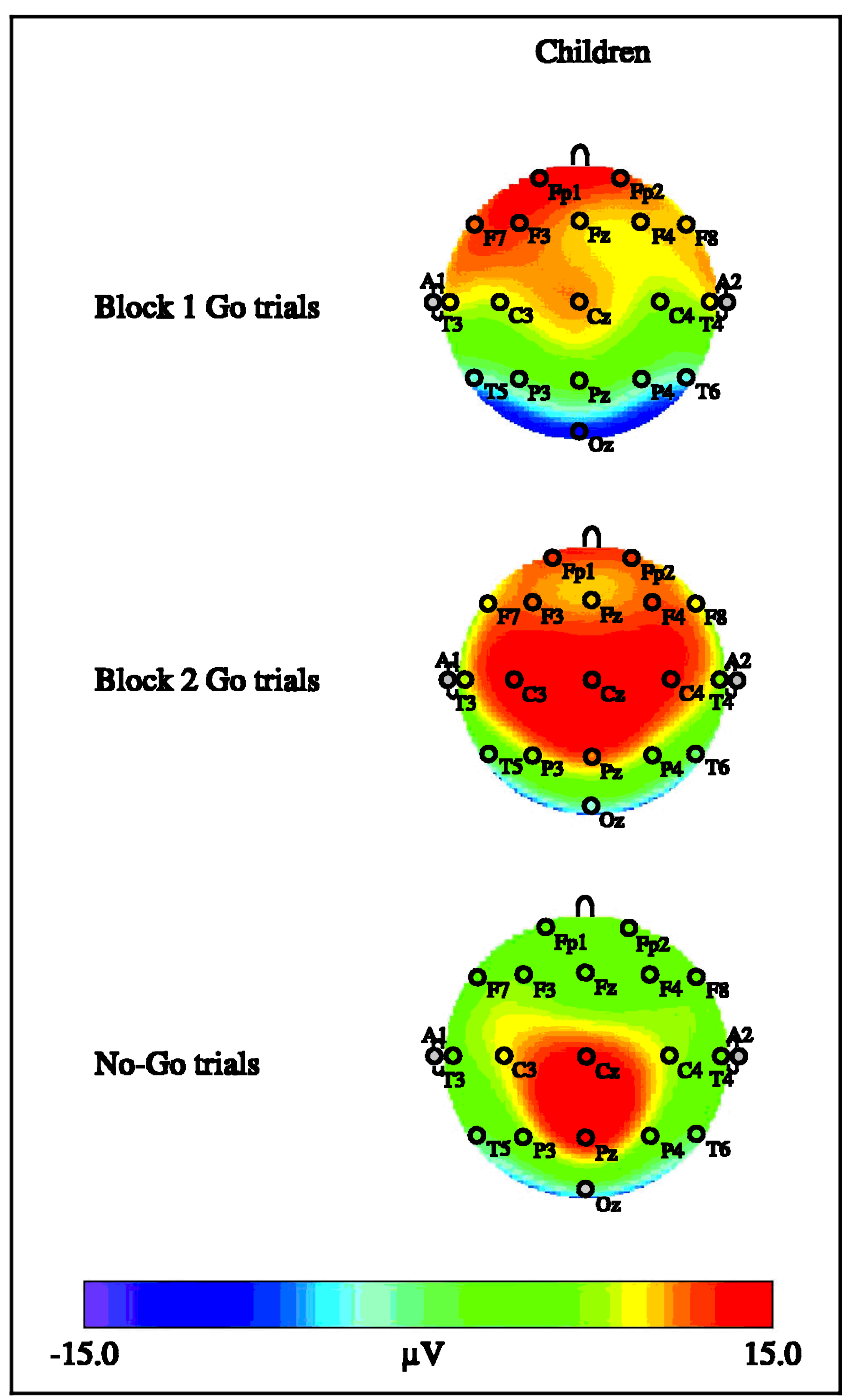

Figure 4. The Laplacian maps of the late frontal component at $900 \mathrm{msec}$ for children.

have contributed to the greater activation during Block 2 . Thus, it may be differences in processing the Block 2 go trials that account for the greater activation seen by children as compared to adults in Block 2 in the fMRI study. These data impact our interpretation of the fMRI study. It seems that the greater activation in frontal regions for children, as compared to adults, was not simply due to the increase in resources needed to inhibit a response to no-go trials. The greater P3 activation may reflect a process involved in the regulation of performance on Block 2 go and no-go trials. However, it is important to note that the children in the current study were 6 to 7 years of age while the children in the fMRI study were 7 to 12 years of age, which may have impacted the results and interpretations of the current findings.

Because of the greater temporal resolution of ERPs, there is additional evidence not available from the fMRI data that children are using qualitatively different pro- cesses than adults on this task. Specifically, as can be seen in Figure 4, a later positive component was present for children at frontal sites. For children, this late component displayed a unique pattern for the no-go trials. That is, the amplitude of this component was smaller in the no-go trials as compared to the Block 1 and Block 2 go trials. This late positive component, therefore, may reflect processes involved in maintaining the inhibition of a motor response. Alternatively, because the component occurs after the response as inferred from mean reaction times, it might reflect processes involved in the evaluation of a response. This late frontally distributed component, which was not present in adults, suggests that children may involve multiple sources in the cognitive processing of this task.

Surprisingly, the amplitude of the N2, thought to be involved in inhibition, was not affected by task condition. Several studies using similar tasks have reported greater amplitude of the N2 in response to trials requiring inhibition of a motor response (Jackson et al., 1999; Overtoom et al., 1998; Eimer, 1993). Although the reason for the null finding in this study is unclear, it should be noted that the task differed from the tasks previously used in ERP studies in multiple ways, such as task complexity and target/non-target ratio. As noted above, it is believed that the N2 may have been influenced by the large proceeding positive component (i.e., the P3), which may have induced an early resolution of the N2.

As expected, reaction times were not related to latency of the ERP components due to the emphasis on speed as well as accuracy on this task (Donchin et al., 1986). However, associations between accuracy and latency and amplitude of the ERP components were not found. Although there is limited evidence for such associations from previous research (Falkenstein et al., 1999; Verbaten et al., 1994), differences in task and subject population may account for variability in findings across these studies.

Overall, the results of this study have several implications. These data suggest that adults and children may be using additional and/or different processes to perform this task. As illustrated by their behavioral performance, this task is significantly more difficult for children than adults. Furthermore, the ERP data indicate that children may be drawing upon additional resources to engage in the cognitive processes necessary to perform on this task. First, the children displayed a different pattern of $\mathrm{P} 3$ activation during Block 2 as compared to adults. Second, the children showed a later frontally distributed component. Age differences in the morphology and topography of the ERP components suggest that multiple sources may be contributing to the neural processing of this task for children. Results of this study illustrate the complementary nature of ERP and fMRI methods for investigating the neural processes involved in a cognitive task. 


\section{METHODS}

\section{Participants}

Participants in the final sample consisted of 20 adults ( $M=22.1$ years, $S D=3.1 ; 9$ women) and 21 six-year-old children $(M=76.4$ months, $S D=3.3 ; 12$ girls $)$, all righthanded and with no known history of visual or neurological problems. An additional 24 adults were tested but excluded from the final sample due to technical errors $(n=5)$, medical history $(n=4)$, or excessive data artifact $(n=15)$. An additional 20 six-year olds were tested but excluded from the final sample due to lefthandedness $(n=1)$, medical history $(n=1)$, or excessive data artifact $(n=18)$. Adult participants were undergraduates who received extra credit in an introductory psychology course for their participation. Child participants were recruited from a list of parents who had previously indicated an interest in participating in developmental research.

\section{Measures}

\section{Behavioral Data}

Button presses and ERPs were recorded while participants completed an inhibitory control task drawn from a battery developed by Casey et al. (1997). This task measures the ability of participants to inhibit a prepotent motor response by selectively attending and responding to target stimuli while ignoring or inhibiting responses to equally salient non-target stimuli (Casey et al., 1997).

On each trial, a single letter was presented in the center of a computer screen. The stimuli were single, uppercase letters in white typeface against a black background. The letters were $1-1 / 8$ in. in height and $1 / 2$ in. in width and subtended a visual angle of $2.65^{\circ}$ and $1.19^{\circ}$. Target stimuli consisted of all letters except the letter "X," while non-target stimuli consisted of the letter "X." Participants held a button box in both hands and were instructed to press a button as quickly as possible to every letter (i.e., target stimuli) except the letter "X" (i.e., non-target stimuli). Prior to the task, child participants were given 10 practice trials in order to ensure they understood the instructions. After each practice trial, children received feedback regarding their performance.

This task contained two blocks of trials: (1) Block 1 consisted of the first 42 trials and contained 100\% targets (i.e., Block 1 go trials) and (2) Block 2 consisted of trials 43 through 84 and contained 50\% target stimuli (i.e., Block 2 go trials) and 50\% non-target stimuli (i.e., no-go trials). That is, the first 42 trials consisted entirely of go trials, while the last 42 trials consisted of go and no-go trials with target and non-target stimuli presented in random order at equal frequencies.

The number of false alarms (i.e., responding to nontarget stimuli) or the number of omissions (i.e., failing to respond to target stimuli) and average reaction time in milliseconds was determined for the Block 1 go, Block 2 go, and no-go trials.

\section{Electroencephalogram (EEG) Recording}

EEG was recorded using Electro-Cap electrode caps with silver-silver-chloride $(\mathrm{Ag}-\mathrm{Ag}-\mathrm{Cl})$ electrodes located over the midline (Fz, Cz, Pz, Oz), left (FP1, F3, F7, C3, P3, T3, T5), and right (FP2, F4, F8, C4, P4, T4, T6) scalp regions according to the International 10-20 System (Jasper, 1958; See Figure 5). Scalp electrodes were recorded referenced to $\mathrm{Cz}$ and then re-referenced to linked mastoids prior to data reduction and analysis. The electrooculogram (EOG) was recorded from bipolar, miniature electrodes placed vertically above and below the right eye. Pre- and posttest impedances were recorded and were considered acceptable when they were below $5 \mathrm{k} \Omega$. A Grass ${ }^{\circledR}$ Model 12 Neurodata Acquisition System was used to collect EEG. A gain of 50 was used for scalp electrodes and a gain of 5 was used for EOG electrodes. The amplifier band pass was $0.1-30 \mathrm{~Hz}$, and a $60-\mathrm{Hz}$ notch filter was engaged. EEG and EOG were sampled every $5 \mathrm{msec}$.

Each trial lasted $1600 \mathrm{msec}$ and consisted of $500 \mathrm{msec}$ of stimulus presentation and then $1100 \mathrm{msec}$ of poststimulus recording. The intertrial interval was $400 \mathrm{msec}$. A black background appeared on the computer screen during poststimulus recording and the intertrial interval. During testing, the room was completely dark except for the illumination of the computer screen.

Prior to averaging, trials with movement artifact (EEG greater than $\pm 100 \mu \mathrm{V}$ ) were rejected. Data were then re-referenced to linked mastoids, and

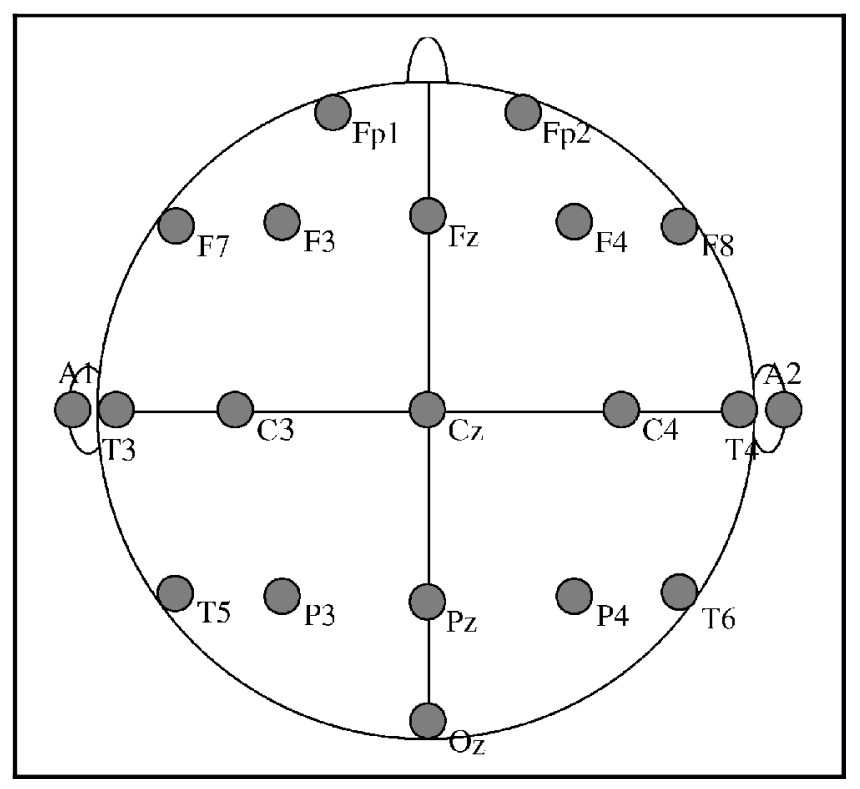

Figure 5. The placement of electrodes in the International 10-20 System. 
eye-movement-related artifact was corrected (Gratton, Coles, \& Donchin, 1983). Using the first $100 \mathrm{msec}$ of stimulus presentation as the baseline, individual trials were baseline corrected and then averaged for each participant within each stimulus type (i.e., Block 1 go, Block 2 go, and no-go trials). For each participant, an equal number of trials were selected for the three stimulus types.

\section{Data Analysis}

ERP components were analyzed by selecting time windows for comparison of components and then computing peak amplitude and latency to peak for each component. Because the morphology of the ERPs for adults and children differed, components were identified relative to the number of major positive and negative peaks following stimulus onset. This necessitated the use of different time windows for comparison of the same component across adults and children. All time window values are relative to stimulus onset, and the peak amplitudes are calculated relative to the baseline amplitude. The peak of the N2 was identified as the maximum negative value occurring between 250 and $470 \mathrm{msec}$ for adults and 300 and $680 \mathrm{msec}$ for children. Based on visual inspection of the ERP waveforms, it was determined that the N2 was maximal at frontal leads for adults and children. Thus, analyses of the N2 were restricted to the frontal leads (Fz, F3, and $\mathrm{F} 4)$. The peak of the $\mathrm{P} 3$ was identified as the maximum positive value between 300 and $700 \mathrm{msec}$ for adults and between 475 and $1090 \mathrm{msec}$ for children. The P3 was analyzed at parietal leads (Pz, P3, and P4) because it was maximal at parietal leads based on visual inspection of the ERP waveforms. Additionally, a late positive component was identified at frontal leads for only children. The peak of the late positive component for children was identified as the maximum positive value between 650 and $1100 \mathrm{msec}$ at frontal leads (Fz, F3, and F4).

Repeated measures analyses of variance (ANOVA) with Greenhouse-Geiser correction for sphericity as needed were conducted to examine the behavioral and ERP data. Post hoc paired $t$ tests using Fisher's LSD were conducted to follow up significant main effects and interactions from the repeated measures ANOVA. The first set of analyses were performed using repeated measures ANOVA to investigate differences between adults and children and across the different type of trials for accuracy and reaction time on the task. The next set of analyses used repeated measures ANOVA to examine differences in peak amplitude and latency to peak of the $\mathrm{N} 2$ and $\mathrm{P} 3$ across age group, task trials, and electrode sites. Additionally, a repeated measures ANOVA was used to examine differences in the maximum amplitude and latency to peak for the late positive component across trials and electrode sites for children. Next, analyses were conducted to explore the relation between performance on the task and the physiological data. Descriptive data and Pearson product correlations were used to explore the relation between reaction times and the latency of the ERP components. Finally, Pearson product correlations were used to investigate the associations between false alarms (i.e., responding to non-target stimuli) and the ERP data.

\section{Acknowledgments}

This research was supported by a grant from the National Institutes of Health (NS329976) to the last author. Portions of this data were presented at the 2000 annual meeting of the Cognitive Neuroscience Society and the 2001 biennial meeting of the Society for Research in Child Development. The authors express their gratitude to the undergraduate students and families who participated in this research project. Thanks are also given to Niki Wenzler and Shanna Newmann for their invaluable assistance with data collection.

Reprint requests should be sent to Charles A. Nelson; Institute of Child Development, University of Minnesota, 51 East River Road, Minneapolis, MN 55455, USA, or via e-mail: canelson@umn.edu.

\section{Notes}

1. For one child, button presses were not recorded due to a technical error. However, behavioral observations indicated that the child was attending to the task. Thus, the child was included in analyses involving ERP data, but not behavioral data.

2. Data from the lead P3 was removed from analyses involving parietal components for one adult due to excessive artifact at that lead.

3. Because children made significantly more errors than adults, particularly on the no-go trials, some of the age differences in the ERP data could have been due to an increase in the number of errors committed by children. Thus, the children who committed 10 or more false alarms $(n=7)$ were removed, and the data were reanalyzed. In general, the pattern of results was consistent. However, several of the post hoc tests were only marginally significant (i.e., $p<.1$ ) with the reduced sample.

\section{REFERENCES}

Carter, C. S., Macdonald, A. M., Botvinick, M. M., Ross, L. L., Stenger, V. A., Noll, D., \& Cohen, J. D. (2000). Parsing executive processes: Strategic vs. evaluative functions of the anterior cingulate cortex. Proceedings of the National Academy of Sciences, U.S.A., 97, 1944-1948.

Casey, B. J., Trainor, R. J., Orendi, J. L., Schubert, A. B., Nystrom, L. E., Giedd, J. N., Castellanos, F. X., Haxny, J. V., Noll, D., Cohen, J. D., \& Rapoport, J. L. (1997). A developmental functional MRI study of prefrontal activation during performance on a go-no-go task. Journal of Cognitive Neuroscience, 9, 835-847.

Chao, L. L., \& Knight, R. T. (1995). Human prefrontal lesions increased distractibility to irrelevant sensory inputs. NeuroReport, 6, 1605-1610.

Chelune, G. J., Ferguson, W., Koon, R., \& Dickey, T. O. (1986). Frontal lobe disinhibition in attention deficit disorder. Child Psychiatry and Human Development, 16, 221-234. 
Cohen, H. L., Porjesz, B., Begleiter, H., \& Wang, W. (1997). Neurophysiological correlates of response production and inhibition in alcoholics. Alcoholism: Clinical and Experimental Research, 21, 1398-1406.

Dempster, F. N. (1992). The rise and fall of the inhibitory mechanism: Toward a unified theory of cognitive development and aging. Developmental Review, 12, $45-75$.

Diamond, A. (1989). The performance of human infants on a measure of frontal cortex function, the delayed response task. Developmental Psychobiology, 22, 271-294.

Diamond, A., \& Goldman-Rakic, P. S. (1989). Comparison of human infants and rhesus monkeys on Piaget's A-not B task: Evidence for dependence on dorsolateral prefrontal cortex. Experimental Brain Research, 74, 24-40.

Donchin, E., Karis, D., Bashiore, T. R., Coles, M. G. H., \& Gratton, G. (1986). Cognitive psychophysiology and human information processing. In M. G. H. Coles, E. Donchin, \& S. W. Porges (Eds.), Psychophysiology: Systems, processes, and applications (pp. 244-267). New York: Guilford Press.

Eimer, M. (1993). Effects of attention and stimulus probability on ERPs in a Go/Nogo task. Biological Psychology, 35, $123-138$.

Falkenstein, M., Hoormann, J., \& Hohnsbein, J. (1999). ERP components in Go/Nogo tasks and their relation to inhibition. Acta Psychologica, 101, 267-291.

Fallgatter, A. J., \& Strik, W. K. (1999). The NoGo anteriorization as a neurophysiological standard-index for cognitive response control. International Journal of Psychophysiology, 32, 233-238.

Gorenstein, E., Mammato, C., \& Sandy, J. (1989). Performance of inattentive-overactive children on selected measures of prefrontal-type function. Journal of Clinical Psychology, 45, 619-632.

Gratton, G., Coles, M. G. H., \& Donchin, E. (1983). A new method of off-line removal of ocular artifact. Electroencephalography and Clinical Neurophysiology, 10, 468-484.

Iversen, S. D., \& Mishkin, M. (1970). Perseverative inference in monkey with following selective lesions of the inferior prefrontal convexity. Experimental Brain Research, 11, $376-386$.
Jackson, S. R., Jackson, G. M., \& Roberts, M. (1999). The selection and suppression of action: ERP correlates of executive control in humans. NeuroReport, 10, 861-865.

Jasper, H. H. (1958). The ten-twenty electrode system of the international federation. Electroencephalography and Clinical Neurophysiology, 10, 371-375.

Keifer, M., Marzinzik, F., Weisbrod, M., Scherg, M., \& Spitzer, M. (1998). The time course of brain activations during response inhibition: Evidence from event-related potentials in a Go/No go task. NeuroReport, 9, 765-770.

Mishkin, M. (1964). Perseveration of central sets after frontal lesions in monkey. In J. M. Warren \& K. Akert (Eds.), The frontal granular cortex and behavior (pp. 219-241). New York: McGraw-Hill.

Overtoom, C. C., Verbaten, M. N., Kemner, C., Kenemans, J. L., van Engeland, H., Buitelaar, J. K., Camfferman, G., \& Koelega, H. S. (1998). Associations between event-related potentials and measures of attention and inhibition in the Continuous Performance Task in children with ADHD and normal controls. Journal of the American Academy of Child and Adolescent Psychiatry, 37, 977-985.

Pardo, J. V., Pardo, J. P., Janer, K. W., \& Raichle, M. E. (1990). The anterior cingulate cortex mediates processing selection in the Stroop attentional conflict paradigm. Proceedings of the National Academy of Sciences, U.S.A., 87, 256-259.

Tekok-Kilic, A., Schucard, J. L., \& Schucard, D. W. (2001). Stimulus modality and Go/NoGo effects on P3 during parallel visual and auditory continuous performance tasks. Psychophysiology, 38, 578-589.

van Leewen, T. H., Steinhausen, H. C., Overtoom, C. C., Pascual-Marqui, R. D., van't Klooster, B., Rothenberger, A., Sergeant, J. A., \& Brandeis, D. (1998). The continuous performance test revisited with neuroelectric mapping: Impaired orienting in children with attention deficits. Behavioral Brain Research, 94, 97-110.

Verbaten, M. N., Overtoom, C. C. E., Koelega, H. S., Swaab-Barneveld, H., van der Gaag, R. J., Buitelaar, J., \& van Engeland, H. (1994). Methylphenidate influences on both early and late ERP waves of ADHD children in a Continuous Performance Test. Journal of Abnormal Child Psychology, 22, $561-578$. 